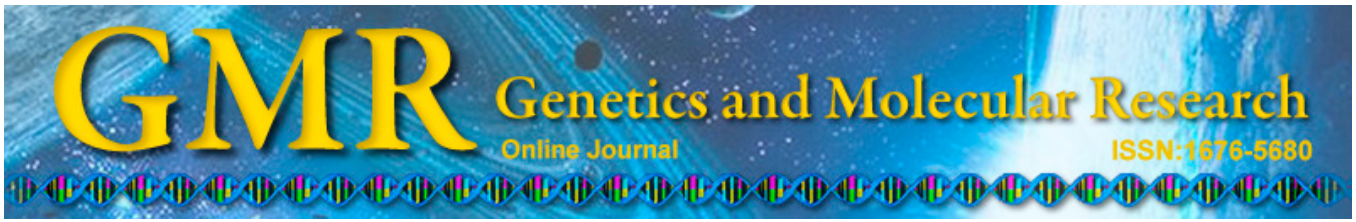

\title{
Development of a new amplification-refractory mutation system for detection of a single nucleotide polymorphism linked to drug resistance in Ancylostoma caninum
}

\author{
L.F. Furtado and É.M. Rabelo \\ Departamento de Parasitologia, Universidade Federal de Minas Gerais, \\ Instituto de Ciências Biológicas, Belo Horizonte, MG, Brasil \\ Corresponding author: É.M. Rabelo \\ E-mail: elidam.rabelo@gmail.com
}

Genet. Mol. Res. 14 (2): 5103-5111 (2015)

Received August 5, 2014

Accepted November 25, 2014

Published May 12, 2015

DOI http://dx.doi.org/10.4238/2015.May.12.13

\begin{abstract}
Single nucleotide polymorphisms at codons 167, 198, and 200 in the $\beta$-tubulin isotype 1 gene have been associated with benzimidazole resistance. Until now, the only mutation observed in Ancylostoma caninum was at codon 200 of this gene. However, the standardized methodologies used to detect mutations in this species are faulty. The objective of this study was to standardize a molecular technique based on amplification-refractory mutation system-polymerase chain reaction (ARMS-PCR) for detecting the mutation at codon 200 in the A. caninum $\beta$-tubulin isotype 1 gene. Controls were synthesized both for the absence of the mutation, using conventional PCR, and for the presence of the mutation, using the Megaprimer-PCR technique. After
\end{abstract}


standardization of the ARMS-PCR using the controls, the technique was validated through an analysis of 75 A. caninum DNA samples, followed by sequencing. The results revealed that the developed technique has high sensitivity, specificity, and reproducibility, which allow its application in the field.

Key words: Drug resistance; ARMS-PCR; Megaprimer-PCR; Beta-tubulin; Ancylostoma caninum

\section{INTRODUCTION}

Ancylostoma caninum is a nematode of great veterinary importance that parasitizes canines and felines (Dias et al., 2013). The primary prophylactic method taken against this parasite, and nematodes in general, is mass drug administration (MDA). Although this strategy ensures that infections are maintained below morbidity levels, periodic MDA can be risky because it induces strong selective pressure for drug-resistant strains (Melo and Bevilaqua, 2005; Prichard et al., 2012).

The most commonly used drugs in this strategy are the benzimidazoles. These drugs block the polymerization of parasite microtubules by binding to $\beta$-tubulin proteins (Lacey, 1988). Single nucleotide polymorphisms (SNPs) in the $\beta$-tubulin isotype 1 gene at codons 167, 198, and 200 have been linked to benzimidazole resistance in some nematodes of importance to humans, including Wuchereria bancrofti, Ascaris lumbricoides, and Trichuris trichiura (Hoti et al., 2003; Diawara et al., 2013), and nematodes of veterinary importance, such as Haemonchus contortus, Teladorsagia circumcincta, and Cooperia oncophora (Elard et al., 1996; Njue and Prichard, 2003; Ghisi et al., 2007). However, until now, there have been no studies demonstrating the presence of mutations in the $\beta$-tubulin isotype 1 gene in A. caninum.

A molecular technique widely used for the detection of these SNPs is based on amplification-refractory mutation system polymerase chain reaction (ARMS-PCR) (Figure 1). This technique relies on the fact that conventional Taq DNA polymerase does not show 3'-5' exonuclease activity, so a mismatched pairing at the 3'-end of the primer with the DNA sample makes amplification impossible (Wang et al., 2014). This technique uses only one pair of primers per reaction, whereas its variant, the tetra-primer ARMS-PCR, uses four primers in the same reaction (two external and two internal primers), one primer to anneal to the non-mutated allele sequence and one primer to anneal to the mutated allele (Ye et al., 2001). Although the tetra-primer ARMS-PCR technique can produce a straightforward result, its standardization, in which four different primers must work together, is not always readily accomplished. A unique mismatch at the 3 '-end of the primer is insufficient to avoid non-specific amplification, making a second mismatch necessary (Schwenkenbecher et al., 2007). Therefore, the objective of this study was to standardize a molecular technique based on ARMS-PCR for the detection of mutation at codon 200 in the $A$. caninum $\beta$-tubulin isotype 1 gene. 

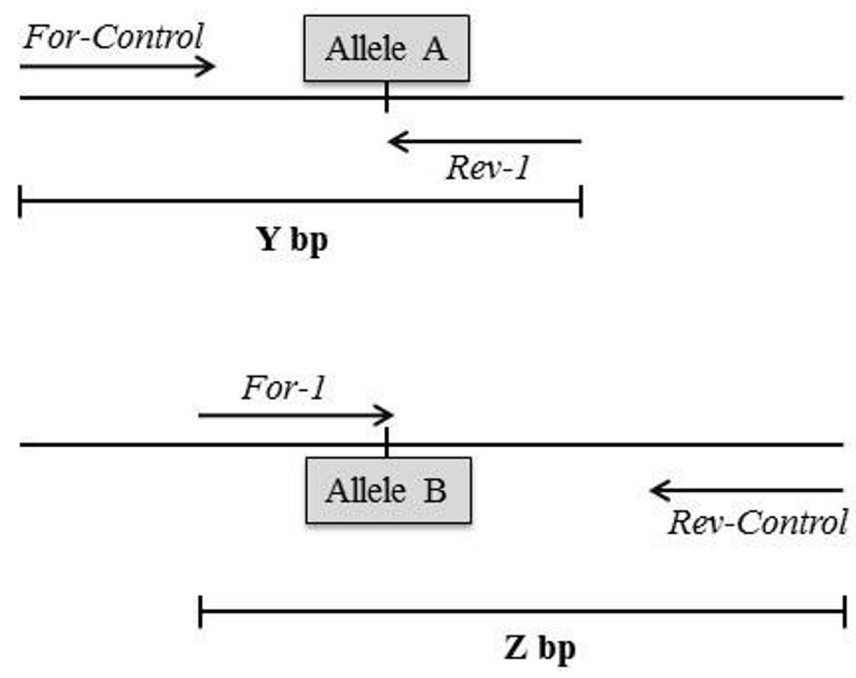

Figure 1. Schematic representation of the amplification-refractory mutation system polymerase chain reaction (ARMS-PCR) for single nucleotide polymorphism (SNP) analysis. The hypothetical allele A is detected by amplification using For-Control and Rev-1 primers ( $\mathrm{Y}$ bp). The hypothetical allele B is detected by the amplicon from primers For-1 and Rev-Control ( $\mathrm{Z}$ bp). For one same sample, it is necessary to perform both reactions. If the individual is heterozygous, amplification occurs in both reactions; if homozygous, only one of the reactions results in generation of an amplified product.

\section{MATERIAL AND METHODS}

\section{Ethics statement}

Adult worms were collected during necropsy of the small intestines of 10 dogs of undefined breeds, which were routinely subjected to euthanasia following the approved procedures of the Municipality Health Centers of the Belo Horizonte by the Zoonoses Control Center (CCZ). These dogs are typically euthanized because they are infected by Leishmania spp. The anesthesia and euthanasia procedures were performed by CCZ professionals, in compliance with current legislation. All animal procedures were approved by the animal-care Ethics Committee of the Federal University of Minas Gerais (Protocol 328/12) and were performed under the guidelines from Conselho Nacional de Controle de Experimentação Animal (CONCEA; Brazilian Council of Animal Experimentation); the procedures strictly followed the Brazilian law for "Procedures for the Scientific Use of Animals" (11.794/2008).

\section{Parasites and DNA extraction}

Adult hookworms were washed in physiological saline, identified morphologically to the species level and frozen $\left(-80^{\circ} \mathrm{C}\right)$ until use. DNA extraction was performed using $75 \mathrm{~A}$. caninum ( 32 males and 43 females), employing a technique described by Waldschmidt et al. (1977). Individual adult worms were ground in a microcentrifuge tube using a glass pestle and liquid $\mathrm{N}_{2}$ and were then homogenized with $400 \mu \mathrm{L}$ lysis buffer [50 mM Tris-HCl, $\mathrm{pH}$ 8.0, 
$2 \%$ sodium dodecyl sulfate, $0.75 \mathrm{M} \mathrm{NaCl}, 10 \mathrm{mM}$ ethylenediaminetetraacetic acid (EDTA)] and $100 \mu \mathrm{g} / \mathrm{mL}$ proteinase $\mathrm{K}$ (Promega, Madison, WI, USA). The samples were subsequently incubated at $65^{\circ} \mathrm{C}$ for $30 \mathrm{~min}$, deproteinized with chloroform:isoamyl alcohol (24:1), and centrifuged at $12,000 \mathrm{~g}$ for $5 \mathrm{~min}$ at $25^{\circ} \mathrm{C}$. The organic phase was transferred to another microcentrifuge tube and incubated with $100 \mu \mathrm{g} / \mathrm{mL}$ RNase A (Ludwig Biotec, Porto Alegre, RS, Brazil) for $30 \mathrm{~min}$ at $37^{\circ} \mathrm{C}$. The samples were next submitted to a second deproteinization step with chloroform:isoamyl alcohol (24:1) and precipitated with $400 \mathrm{~mL}$ isopropanol after centrifugation at $12,000 \mathrm{~g}$ for $30 \mathrm{~min}$ at $4^{\circ} \mathrm{C}$. The resulting pellet was resuspended in $15 \mu \mathrm{L}$ DNase-free double-distilled water.

\section{Primer design}

The primers employed in this study were designed using the program Oligo Explorer 1.4 (Gene Link, Hawthorne, NY, USA), based on the sequence of the $\beta$-tubulin isotype 1 gene of A. caninum available in the National Center for Biotechnology Information database (www. ncbi.nlm.nih.gov) under accession number DQ459314.1. Table 1 lists all of the primers, the primer annealing temperatures, and, when applicable, the position of the base that was modified. Table 2 shows the amplicon sizes for the different primer combinations.

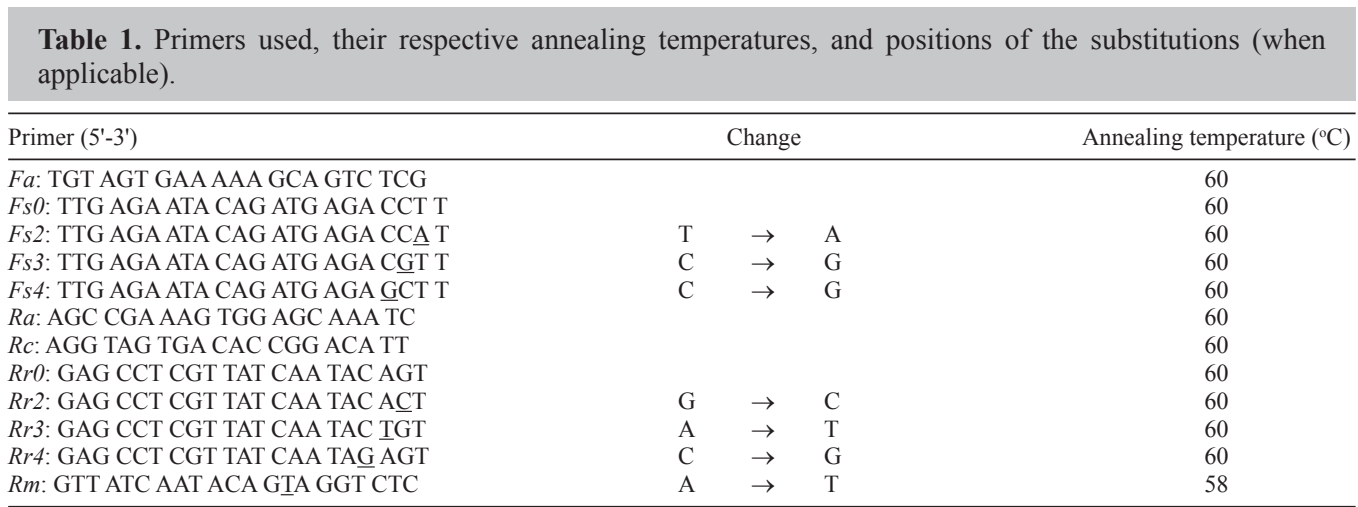

Positions where changes have been made are underlined.

Table 2. Primer combinations and their amplicon sizes.

\begin{tabular}{lc}
\hline Primer combination & Size of amplicon (bp) \\
\hline$F a+R a$ & 441 \\
$F a+R c$ & 331 \\
$F a+R r 0$ & 164 \\
$F a+R r 2$ & \\
$F a+R r 3$ & \\
$F a+R r 4$ & 157 \\
$F a+R m$ & 209 \\
$F s 0+R c$ & \\
$F s 2+R c$ & \\
$F s 3+R c$ & \\
$F s 4+R c$ & \\
\hline
\end{tabular}




\section{Synthesis of negative and positive controls}

The ARMS-PCR technique was used to detect mutations at codon 200. To standardize this molecular technique, we first synthesized controls for the presence and absence of the mutation. PCR amplifications were performed using GoTaq Green Master Mix (Promega), with a final concentration of $0.2 \mu \mathrm{M}$ for each primer and approximately $20 \mathrm{ng}$ DNA. All amplification steps were performed in a Mastercycler (Eppendorf, Hamburg, Germany) thermocycler according to the following program: $94^{\circ} \mathrm{C}$ for $5 \mathrm{~min}$ for the initial denaturation of double-stranded DNA, followed by 30 cycles at $94^{\circ} \mathrm{C}$ for $1 \mathrm{~min}, 60^{\circ} \mathrm{C}$ for $1 \mathrm{~min}$, and $72^{\circ} \mathrm{C}$ for $1 \mathrm{~min}$, with a final extension step at $72^{\circ} \mathrm{C}$ for 8 min. In all amplification runs, a "blank" sample was included in which the DNA was replaced with water to assess the presence of possible contaminants.

To synthesize the negative control for the mutated allele at codon 200, hereafter referred to as $T u b N$, an initial PCR amplification was performed with the primers $F a$ and $R a$ using genomic DNA from A. caninum. Next, semi-nested PCR was performed using the primers $F a$ and $R c$, and the obtained fragment was sequenced to confirm the absence of mutation. The fragment was subsequently cloned using the pGEM-T Easy Vector System (Promega), then transformed into XL1-blue cells (Phoneutria Bio, Santa Branca, SP, Brazil) and recovered via miniprep (Wizard Plus Miniprep DNA Purification System, Promega).

To synthesize the mutated positive control, referred to as TubM, site-directed mutagenesis was performed using the Megaprimer-PCR technique. The cloned TubN was employed as a template for PCR amplification using the primer combination of $F a$ and $R m$. The $R m$ primer was designed to include a mismatch at position 8 of the $3^{\prime}$-end of the primer that replaced an A nucleotide with a $\mathrm{T}$ to mimic the mutated sequence. The reaction product was subjected to electrophoresis on a $1.0 \%$ agarose gel (w/v) (Midsci, St. Louis, MO, USA) with $0.5 \mathrm{X}$ Trisacetate EDTA (TAE) buffer, and the gel was stained with GelRed (Biotium, Hayward, CA, USA). The fragment was then excised from the gel and purified (Illustra GFX PCR DNA and Gel Band Purification Kit, GE Healthcare, Little Chalfont, UK), and the concentration was determined. Approximately $25.0 \mathrm{ng}$ first reaction product was used as a forward mega-primer in the second reaction, in combination with the reverse $R c$ primer. The product of this reaction was purified, sequenced, cloned, and recovered in the same way as described for TubN.

\section{ARMS-PCR}

The synthesized TubN and TubM controls were used in PCR assays to test and select the primers that were synthesized. For each control, PCRs were performed with different combinations of primer pairs. Reactions combining primer pairs consisting of $F a$ and different reverse primers were performed to detect the mutated fragment, whereas different combinations of forward primers in association with the $R c$ reverse primer were applied to detect the fragment without a mutation. To determine whether the correct annealing of the last bases in the target sequence by itself would be sufficient for the high specificity of such conditions, the primers $F s$ and $R r$ were designed without any mismatches. Based on the possibility that these primers might not distinguish fragments with or without mutations, primers with a mismatch at different positions were designed to ensure high specificity. Thus, mismatch primers were designed at position 2 of the $3^{\prime}$-end $\left(F_{s} 2\right.$ and $R r 2$ ), and at positions 3 ( $F_{s} 3$ and $R r 3$ ) and 4 (Fs4 and Rr4). The base pair substitutions were designed as described by Ye et al. (2001). The products of these reactions were subjected to electrophoresis on $1.0 \%$ agarose gels $(\mathrm{w} / \mathrm{v})$ with 
0.5X TAE buffer, and the gels were stained with GelRed.

\section{Validation of ARMS-PCR}

After the synthesis of the controls and the selection of primers, the technique was validated by use of 75 DNA samples from $A$. caninum. A first PCR amplification with primers $F a$ and $R a$ was performed for each sample, after which a second reaction was carried out using the selected primers $F_{s} 4+R c$ and $F a+R r 4$. These samples also were subjected to sequencing. Thus, the product of the first reaction with primers $F a$ and $R a$ was used for a second reaction in a final volume of $25 \mu \mathrm{L}$, applying the same conditions described for the control syntheses, with a $0.2 \mu \mathrm{M}$ concentration of the $F a$ and $R c$ primers. The PCR products were subsequently purified, quantified, and sequenced. Sequencing was performed according to the method originally described by Sanger et al. (1977). All sequencing reactions were carried out using the DYEnamic ET dye terminator cycle sequencing kit (GE Healthcare), and the runs were performed using the MegaBACE Long Read Matrix (GE Healthcare) with the same primers as in the second reaction. Chromatograms were analyzed using the Sequence Scanner software (Life Technologies, Carlsbad, CA, USA).

\section{RESULTS AND DISCUSSION}

Based on the primer analyses, we determined that primers without a second mismatch were non-specific, resulting in amplification regardless of the plasmid that was used, either with primers directed to the control (Figure 2A, lanes 1 and 2) or when primers to the mutated allele where used (Figure 2B, lanes 1 and 2).
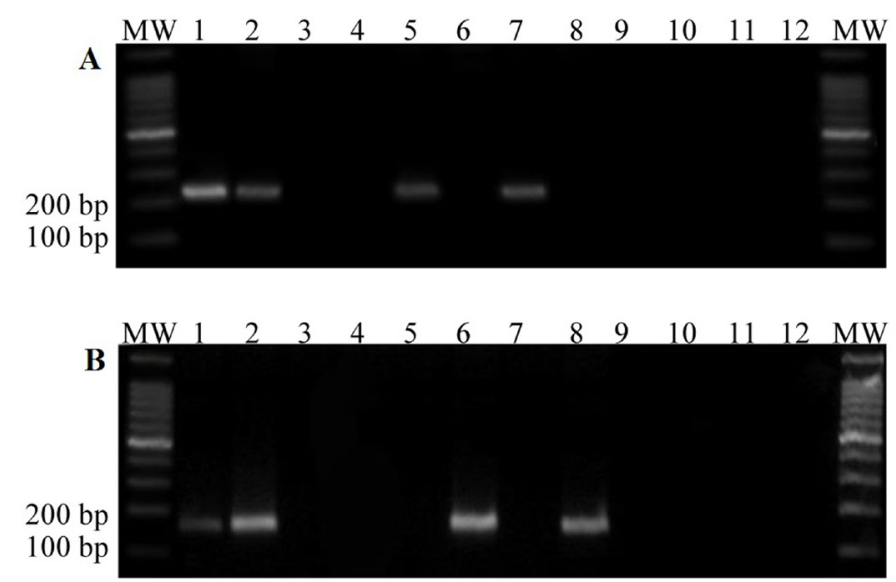

Figure 2. Electropherograms of primer selection reactions. A. Selection of the forward primer for the non-mutated allele. Lanes 1 and 2: the polymerase chain reaction (PCR) product resulting from the combination of primers $F s$ and $R c$; lanes 3 and 4: primers $F s 2$ and Rc; lanes 5 and 6: primers $F s 3$ and $R c$; and lanes 7 and 8: primers $F s 4$ and $R c$. B. Selection of the reverse primer for the mutated allele. Lanes 1 and 2: the PCR product resulting from the combination of primers $F a$ and $R r$; lanes 3 and 4: primers $F a$ and $R r 2$; lanes 5 and 6: primers $F a$ and $R r 3$; and lanes 7 and 8: primers $\mathrm{Fa}$ and $\mathrm{Rr} 4$. For the two gels, the TubN negative (wild type) control was used for the reactions in the odd-numbered lanes, and the mutated positive control TubM was used in the even-numbered lanes. Lanes 9-12 correspond to the blank control for the reactions. The images show agarose gels (1\%) stained with gel red. MW: 100-bp molecular weight marker. 
In contrast, primers with a mismatch at position 2 of the 3 '-end were unable to anneal or produce any amplification product (Figure 2A, B, lanes 3 and 4). Primers with a mismatch at positions 3 and 4 were specific (Figure 2A, B, lanes 5-8). Therefore, we chose to use the forward primer $F_{s} 4$ (for annealing in the absence of a mutation) and the reverse primer $\operatorname{Rr} 4$ (for annealing in the presence of a mutation), as these primers presented a mismatch at the farthest position from the 3 '-end. Figure 2 shows the results of PCR amplification using the forward (A) and reverse (B) primer selections.

The standardization of molecular techniques is not always simple, and the extrapolation of species-specific protocols may not always be successful. For example, in analyzing codon 200 in Necator americanus, Albonico et al. (2004) did not add a second mismatch in the primer, whereas Ye et al. (2001) reported the need to add a mismatch at position 2 of the 3 '-end of the primer. These data contradict the results obtained in the present study. We found that primers without a mismatch did not show good specificity and annealed to the target sequences irrespective of whether they included a mutation. Our primers with a mismatch at position 2 of the 3 '-end did not allow annealing, regardless of the target sequence. Our results were satisfactory only when we used primers with a mismatch at the 3rd or 4th position of the 3 '-end. In this study, we attempted to establish a tetra-primer ARMS-PCR approach to simultaneously analyze the two different alleles using four primers in a single reaction; however, the reaction presented low specificity and produced many more fragments than expected, despite all efforts to optimize the reaction, demonstrating that the problem was intrinsic to the region where the primers needed to anneal. Therefore, we opted to perform separate reactions that proved to be completely reproducible.

Based on the standardization of the technique using control plasmids, we validated the method using 75 DNA samples from A. caninum. PCR amplifications were performed in two separate reactions for all of the samples analyzed, thereby allowing both alleles to be assayed separately. In one reaction, a primer set targeting the mutant allele was used, and the other employed a primer set for the non-mutant allele. Of the total samples analyzed, only one sample showed amplification in both reactions (i.e., was heterozygous), while all other reactions resulted in amplification only in the reaction to detect the non-mutated allele. Figure 3 shows a representative gel of PCR products used to validate the technique.

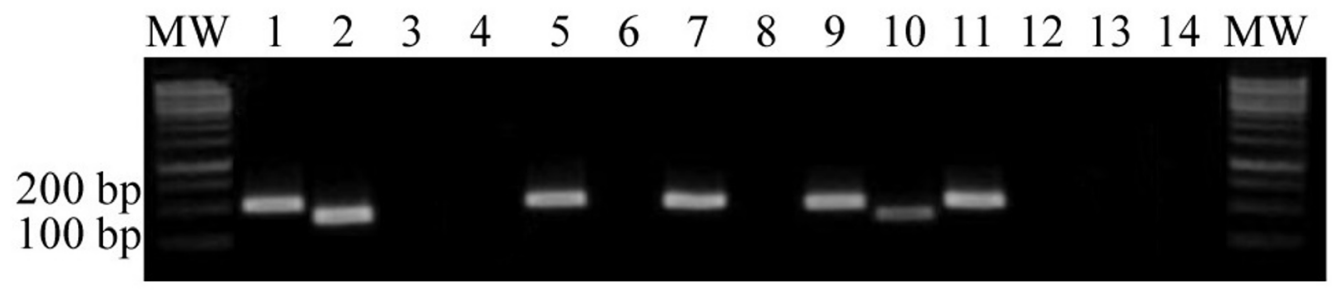

Figure 3. Representative results of the validation of amplification-refractory mutation system polymerase chain reaction (ARMS-PCR). Individual worm genomic DNA samples were subjected to PCR amplification with two sets of primers. The lanes indicated by odd numbers correspond to PCR products obtained using the primer combination $F s 4$ and $R c$ to detect the fragment without a mutation (209 bp), whereas the lanes identified by even numbers correspond to PCR products obtained using the primer combination $\mathrm{Fa}$ and $\mathrm{Rr} 4$ to detect the fragment with a mutation (164 bp). In lanes 1-4, synthesized controls were used (lanes 1 and 4: TubN (negative (wild type) control; and lanes 2 and 3: TubM (mutated positive control)). For each sample, the two reactions were analyzed side by side. Lanes 13 and 14 correspond to the blank controls for the reactions. The image shows an agarose gel (1\%) stained with gel red. MW: 100-bp molecular weight marker. 
The results obtained using these standardized molecular techniques were confirmed through sequencing. Real-time quantitative PCR for the detection of $A$. caninum SNPs had been previously proposed (Schwenkenbecher et al., 2007; Schwenkenbecher and Kaplan, 2009); however, this method requires special equipment. In the present study, a conventional thermal cycler was sufficient to implement ARMS-PCR, and the results could be obtained directly, making them less subject to interpretation errors. Therefore, because the sequencing results confirmed the ARMS-PCR results, the standardized molecular technique described herein represents a reproducible method for detecting mutations at codon 200.

The technique presented in this report has an internal quality control aspect as well, because the samples should be amplified by at least one combination of primers utilized, either to the mutated allele or to the wild-type, drug-sensitive allele. Therefore, PCR was not performed for additional constitutively expressed genes, as such controls were not required. In this study, worms of both sexes were used in the DNA extraction procedure and allelic analyses, but for a screening study, only male worms should ideally be used because egg DNA in the female uterus may affect the results.

Assays for detecting resistance in parasites primarily rely on phenotypic analyses, which are widely applied in livestock (Lyndal-Murphy et al., 2014). Standardization of this molecular tool is a critical advance, particularly because the phenotypic tests offer low sensitivity, as a greater number of excreted eggs in dog feces does not necessarily indicate a higher parasite load. Depending on the degree of agglomeration in the host, A. caninum can modulate oviposition (Krupp, 1961). Thus, diagnoses of helminth resistance in dogs based only on counts of the number of eggs before and after treatment may not indicate the actual levels of resistance.

We conclude that this standardized molecular technique corresponds to a sensitive, specific, and cost-effective method, which may be used for field studies. This technique can assist in monitoring populations of resistant hookworms. The more quickly this monitoring is performed, the greater the chances of delaying the establishment of a parasite population that does not respond to treatment with benzimidazoles, increasing, in turn, the lifespan of these products.

\section{ACKNOWLEDGMENTS}

Research supported by Conselho Nacional de Desenvolvimento Científico e Tecnológico (CNPq; \#470968/2014-1), Fundação de Amparo à Pesquisa do Estado de Minas Gerais (FAPEMIG) (process \#APQ-01924-13), and Pró-Reitoria de Pesquisa da Universidade Federal de Minas Gerais (PRPq/UFMG). Coordenação de Aperfeiçoamento de Pessoal de Nível Superior (CAPES) funded L.F.V. Furtado with a scholarship. We thank the people from the Zoonosis Control Center (CCZ) of Belo Horizonte for their cooperation and help in obtaining the worms.

\section{REFERENCES}

Albonico M, Wright V and Bickle Q (2004). Molecular analysis of the beta-tubulin gene of human hookworms as a basis for possible benzimidazole resistance on Pemba Island. Mol. Biochem. Parasitol. 134: 281-284.

Dias SR, Cunha DE, da Silva SM, Dos Santos HA, et al. (2013). Evaluation of parasitological and immunological aspects of acute infection by Ancylostoma caninum and Ancylostoma braziliense in mixed-breed dogs. Parasitol. Res. 112: 2151-2157.

Diawara A, Halpenny CM, Churcher TS, Mwandawiro C, et al. (2013). Association between response to albendazole treatment and $\beta$-tubulin genotype frequencies in soil-transmitted helminths. PLoS Negl. Trop. Dis. 7: e2247.

Elard L, Comes AM and Humbert JF (1996). Sequences of beta-tubulin cDNA from benzimidazole-susceptible and -resistant

Genetics and Molecular Research 14 (2): 5103-5111 (2015)

CFUNPEC-RP www.funpecrp.com.br 
strains of Teladorsagia circumcincta, a nematode parasite of small ruminants. Mol. Biochem. Parasitol. 79: 249-253.

Ghisi M, Kaminsky R and Mäser P (2007). Phenotyping and genotyping of Haemonchus contortus isolates reveals a new putative candidate mutation for benzimidazole resistance in nematodes. Vet. Parasitol. 144: 313-320.

Hoti SL, Subramaniyan K and Das PK (2003). Detection of codon for amino acid 200 in isotype 1 beta-tubulin gene of Wuchereria bancrofti isolates, implicated in resistance to benzimidazoles in other nematodes. Acta Trop. 88: 77-81.

Krupp IM (1961). Effects of crowding and of superinfection on habitat selection and egg production in Ancylostoma caninum. J. Parasitol. 47: 957-961.

Lacey E (1988). The role of the cytoskeletal protein, tubulin, in the mode of action and mechanism of drug resistance to benzimidazoles. Int. J. Parasitol. 18: 885-936.

Lyndal-Murphy M, Swain AJ and Pepper PM (2014). Methods to determine resistance to anthelmintics when continuing larval development occurs. Vet. Parasitol. 199: 191-200.

Melo ACFL and Bevilaqua CML (2005). Abordagem genética da resistência anti-helmíntica em Haemonchus contortus. Rev. Port. Cienc. Vet. 100: 141-146.

Njue AI and Prichard RK (2003). Cloning two full-length beta-tubulin isotype cDNAs from Cooperia oncophora, and screening for benzimidazole resistance-associated mutations in two isolates. Parasitology 127: 579-588.

Prichard RK, Basáñez MG, Boatin BA, McCarthy JS, et al. (2012). A research agenda for helminth diseases of humans: intervention for control and elimination. PLoS Negl. Trop. Dis. 6: e1549.

Sanger F, Nicklen S and Coulson AR (1977). DNA sequencing with chain-terminating inhibitors. Proc. Natl. Acad. Sci. U.S.A. 74: 5463-5467.

Schwenkenbecher JM and Kaplan RM (2009). Real-time PCR assays for monitoring benzimidazole resistance-associated mutations in Ancylostoma caninum. Exp. Parasitol. 122: 6-10.

Schwenkenbecher JM, Albonico M, Bickle Q and Kaplan RM (2007). Characterization of beta-tubulin genes in hookworms and investigation of resistance-associated mutations using real-time PCR. Mol. Biochem. Parasitol. 156: 167-174.

Waldschmidt AM, Salomão TMF, Barros EG and Campos LAO (1997). Extraction of genomic DNA from Melipona quadrifasciata (Hymenoptera: Apidae, Meliponinae). Braz. J. Genet. 20: 421-423.

Wang ZN, Li MJ, Lan XY, Li MX, et al. (2014). Tetra-primer ARMS-PCR identifies the novel genetic variations of bovine HNF-4 $\alpha$ gene associating with growth traits. Gene 546: 206-213.

Ye S, Dhillon S, Ke X, Collins AR, et al. (2001). An efficient procedure for genotyping single nucleotide polymorphisms. Nucleic Acids Res. 29: e88. 